\title{
Numerical simulation of the non-regular mode of cooling a high-temperature metal billet by the flow of a gas-liquid medium
}

\author{
Sergey Makarov ${ }^{1, *}$, Vyacheslav Dement'yev', Tat'yana Makhneva ${ }^{1}$, and Elena Makarova² \\ ${ }^{1}$ Federal State Budgetary Institution of Sciences «Udmurt Federal Research Centre Ural Branch of the \\ Russian Academy of Sciences», 34, T. Baramzinoy St., Izhevsk, 426067, Russia \\ ${ }^{2}$ Kalashnikov Izhevsk State Technical University, 7, Studencheskaya St., Izhevsk, 426069, Russia
}

\begin{abstract}
A mathematical model of heat transfer at cooling a hightemperature metal billet from structural steel by the flow of a gas-liquid medium in a vertical circular channel is presented. The model has been built with the use of the continuum mechanics approaches and the theory of heat-mass transfer. The non-regular mode of cooling is considered. The results of the numerical parametric investigations of the heat transfer at cooling a metal billet are obtained for a standard regime of thermomechanical strengthening on the basis of the mathematical model of conjugate heat transfer in a two-dimensional nonstationary formulation accounting for the symmetry of the cooling medium flow relative to the longitudinal axis of a cylinder. The control volume approach is used for solving the system of differential equations. The flow field parameters are computed by an algorithm SIMPLE. For the iterative solution of the systems of linear algebraic equations the Gauss-Seidel method with underrelaxation is used. Taking into account evaporation in the liquid, the intensity of the change of the rate of cooling the material of the metal cylindrical billet by the laminar gas-liquid flow is analyzed depending on the time of cooling and the velocity of the gas-liquid flow.
\end{abstract}

\section{Introduction}

The scientific justification of the regularities of heat transfer at non-regular modes of cooling high-temperature metal bodies by gas-liquid media flows is an important problem. Numerous investigations are dedicated to the regularities of the heat-mass transfer processes. In the present paper, the problem is considered on the basis of a mathematical model of conjugate heat transfer. In [1], a mathematical model of conjugate heat transfer in a heterogeneous system "solid - gas-liquid medium" is proposed. The two-dimensional temperature field in the liquid and the solid was determined at the fulfilment of the thermal boundary conditions (IV) at the boundary of the conjugate media. The evaporation in the liquid was evaluated near the surface of the high-temperature cylindrical metal body cooled

\footnotetext{
* Corresponding author: ssmak15@mail.ru
} 
by the longitudinal water flow. The heat transfer at cooling a high-temperature metal cylinder from structural steel by the flow of a gas-liquid medium was investigated with the use of a numerical algorithm [2, 3]. In [4], the flow round a high-temperature solid metal cylinder with a variable cross-section by a gas-liquid medium is considered. The results of the numerical calculations of hydrodynamic parameters of the gas-liquid flow at cooling the metal cylinder were obtained with taking into account evaporation in the liquid. The temperatures, flow velocities, distribution of the vapour concentration were determined in the calculation domain of the circular channel. The regularities of the change of the metal cylinder surface temperature were obtained depending on the gas-liquid medium flow velocity. In [5], the results of the numerical simulation are presented for heat transfer at cooling of a metal billet from steel $30 \mathrm{HGCN} 2 \mathrm{~A}$. The velocities of cooling the billet material by the gas-liquid medium flow in a horizontal circular channel were determined. The regularities of the change in the intensity of cooling were obtained depending on the velocity of the billet movement in the direction opposite to the direction of the cooling medium flow. The numerical simulation results obtained on the basis of a mathematical model of conjugate heat transfer between a gas-liquid medium flow and a metal cylinder in the two-dimensional nonstationary formulation taking into account the symmetry of the cooling medium flow relative to the cylinder longitudinal vertical axis are presented in [6]. The numerical simulation results were verified by comparing with the nature experiment results.

\section{Problem statement}

The objective of the present paper is the creation of a mathematical model of heat transfer at cooling a high-temperature metal billet from structural steel by a gas-liquid medium in a vertical circular channel and the parametric investigation of the change of the velocity of cooling the metal billet material at the non-regular mode of cooling.

As in [7], the metal billet is presented in the form of a solid cylinder of radius $r_{m}$ and length $L$ having an initial temperature $\mathrm{T}_{\mathrm{m} 0}$; the billet is cooled by a liquid flow moving in the $x$-axis direction in an annular space $r_{l}-r_{m}$. The liquid flow has the initial velocity $u_{0}$ and temperature $T_{l o}$. The outer metal ring of radius $r_{m l}$ has the temperature $T_{m l}$. The physical and calculation scheme of the computational domain are presented in Fig 1.

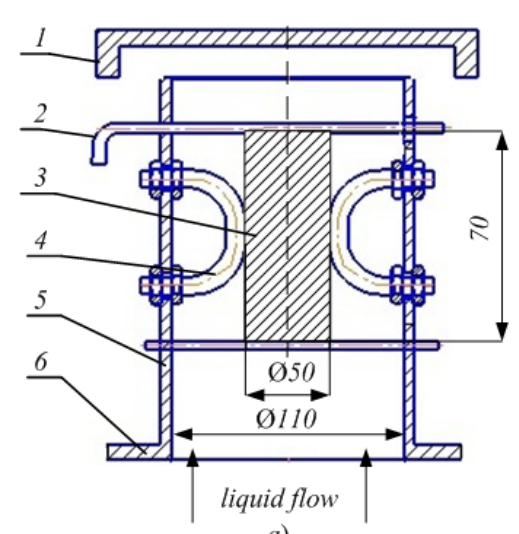

a)

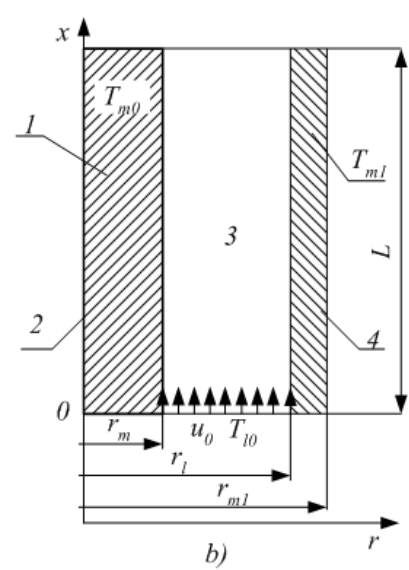

b)

Fig. 1. Physical (a) and calculation (b) scheme of the computational domain: a) 1 - cover; 2 fastening element; 3 - metal billet; 4 - directrices; 5 - tube; 6 - end cap; b) 1 - metal cylinder; 2 cylinder axis; 3 - liquid flow; 4 - outer wall 


\section{Mathematical model}

$$
\begin{aligned}
& \rho \frac{\partial u}{\partial t}+\rho u \frac{\partial u}{\partial x}+\rho v \frac{\partial u}{\partial r}=-\rho g-\frac{\partial p}{\partial x}+\frac{\partial}{\partial x} \mu \frac{\partial u}{\partial x}+\frac{1}{r} \frac{\partial}{\partial r} r \mu \frac{\partial u}{\partial r} \\
& \rho \frac{\partial v}{\partial t}+\rho u \frac{\partial v}{\partial x}+\rho v \frac{\partial v}{\partial r}=-\frac{\partial p}{\partial r}+\frac{\partial}{\partial x} \mu \frac{\partial v}{\partial x}+\frac{1}{r} \frac{\partial}{\partial r} r \mu \frac{\partial v}{\partial r}-\mu \frac{v}{r^{2}} \\
& \frac{\partial \rho}{\partial t}+\frac{\partial(\rho u)}{\partial x}+\frac{1}{r} \frac{\partial(r \rho v)}{\partial r}=0 \\
& \rho c \frac{\partial T}{\partial t}+\rho c u \frac{\partial T}{\partial x}+\rho c v \frac{\partial T}{\partial r}=\frac{\partial}{\partial x} \lambda \frac{\partial T}{\partial x}+\frac{1}{r} \frac{\partial}{\partial r} r \lambda \frac{\partial T}{\partial r}-\dot{m}_{v} Q_{v}-\rho g u \\
& \rho \frac{\partial Y_{v}}{\partial t}+\rho u \frac{\partial Y_{v}}{\partial x}+\rho v \frac{\partial Y_{v}}{\partial r}=\dot{m}_{v}
\end{aligned}
$$

Here, the specific mass velocity of evaporation is $\dot{m}_{v}=(\rho c \Delta T) /\left(Q_{v} \Delta t\right)$, where $\Delta T=\left\{\begin{array}{l}0, \text { if } T(t+\Delta t)<T_{S} \\ {\left[T(t+\Delta t)-T_{n}(t)\right], \text { if } T(t+\Delta t)>T_{S}}\end{array}\right.$ - the liquid overheating; $T_{n}(t)=\max \left[T(t) ; \quad T_{s}\right]$.

$\Delta T$ value can be either positive or negative. In the first case, the the amount of heat energy requied for the 'liquid-vapor' phase transition is taken into account. In the second case, the heat effect produced by the 'vapor-liquid' condensation is taken into consideration provided that there is vapor in the medium, i.e. $Y_{v}>0$. Thus, the energy heat-mass transfer balance of the system is regulated.

The energy equation for the metal cylinder and the outer metal ring is

$$
\rho_{m} c_{m} \frac{\partial T_{m}}{\partial t}=\frac{\partial}{\partial x} \lambda_{m} \frac{\partial T_{m}}{\partial x}+\frac{1}{r} \frac{\partial}{\partial r} r \lambda_{m} \frac{\partial T_{m}}{\partial r}
$$

The effectictive thermophysical parameters of the gas-liquid medium are determined according to $[10]: \rho=\sum_{2} \rho_{i} Y_{i} ; \quad c=\sum_{2} c_{i} \rho_{i} Y_{i} / \rho ; \quad \lambda=\frac{1}{2}\left[\sum_{2} Y_{i} \lambda_{i}+\left(\sum_{2} \frac{Y_{i}}{\lambda_{i}}\right)^{-1}\right]$ $\mu=\frac{1}{2}\left[\sum_{2} Y_{i} \mu_{i}+\left(\sum_{2} \frac{Y_{i}}{\mu_{i}}\right)^{-1}\right] ; \sum_{2} Y_{i}=1$

The initial conditions : $u=u_{0}, v=0, T_{l}=T_{l 0}, T_{m}=T_{m 0}, T_{m l}=T_{l 0}$.

The boundary conditions : $x=0: 0<r<r_{m}, \quad \frac{\partial T}{\partial x}=0$;

$$
r_{m}<r<r_{l}, \quad T=T_{l 0}, u=u_{0}, v=0, Y_{v}=0, \frac{\partial p}{\partial x}=0
$$




$$
\begin{gathered}
r_{l}<r<r_{m 1}, \quad \frac{\partial T}{\partial x}=0 \\
x=L: 0<r<r_{m} \quad \frac{\partial T}{\partial x}=0 ; r_{m}<r<r_{m 1} \quad \frac{\partial T}{\partial x}=0 \\
r_{m}<r<r_{l} \quad \frac{\partial T}{\partial x}=0, \frac{\partial u}{\partial x}=0, \frac{\partial v}{\partial x}=0, \frac{\partial Y_{v}}{\partial x}=0, p=0 \\
0<x<L: r=0 \quad \frac{\partial T}{\partial r}=0 \\
r=r_{m},-\lambda_{m} \frac{\partial T_{m}}{\partial r}=-\lambda \frac{\partial T_{l}}{\partial r}, T_{m}=T_{l}, u=0, v=0, \frac{\partial Y_{v}}{\partial r}=0 \\
r=r_{l} \quad-\lambda_{m 1} \frac{\partial T_{m 1}}{\partial r}=-\lambda_{l} \frac{\partial T_{l}}{\partial r}, T_{m l}=T_{l}, u=0, v=0, \frac{\partial Y_{v}}{\partial r}=0 \\
r=r_{m 1}: \quad \frac{\partial T}{\partial r}=0 .
\end{gathered}
$$

The local cooling rate in the metal billet volume is determined as follows :

$$
V_{m}=\frac{\left|T_{m}(t+\Delta t)-T_{m}(t)\right|}{\Delta t}
$$

In the equations, the following symbols are used: $\boldsymbol{c}$ - specific heat capacity, $\mathrm{J} /(\mathrm{kg} \cdot \mathrm{grad}) ; \boldsymbol{\rho}$ density, $\mathrm{kg} / \mathrm{m}^{3} ; \boldsymbol{\lambda}$ - heat conduction, $\mathrm{W} /(\mathrm{m} \cdot \mathrm{grad}) ; \boldsymbol{\mu}$ - dynamic viscosity, $\mathrm{Pa} \cdot \mathrm{s} ; \boldsymbol{p}$-pressure, $P a ; \boldsymbol{T}$ - temperature, $K ; \boldsymbol{t}$ - time, $s ; \boldsymbol{r}$ - radial coordinate, $m ; \boldsymbol{x}$ - longitudinal coordinate, $m$; $\boldsymbol{L}$ - computational domain length, $m ; \boldsymbol{u}$ - velocity component along $x ; \boldsymbol{v}$ - velocity component along $r, \mathrm{~m} / \mathrm{s} ; \boldsymbol{Y}$ - volume concentration, $\boldsymbol{Q}$ - specific heat of vaporization, $\mathrm{J} / \mathrm{kg}$. Indices: $\boldsymbol{m}$ - metal, $\boldsymbol{l}$-liquid, $\boldsymbol{v}$ - vapor, $\boldsymbol{0}$ - initial value, $\boldsymbol{s}$ - saturation parameters.

\section{Solution procedure}

The control volume approach is used for solving the system of differential equations (1) (6). The flow field parameters (1) - (3) are calculated by an algorithm SIMPLE used for liquid flow simulations. The differential equations are reduced to the system of linear algebraic equation and solved iteratively by the Gauss-Seidel method with the use of the under-relaxation factor. For the calculations, a mesh is used which is condensed near the conjugate boundary 'metal cylinder - liquid' and 'liquid - outer metal wall' on the side of the liquid and metal. Equation (7) is solved after the temperature values have been found at each time step.

\section{Numerical calculation results}

Let us take initial data for our calculation: the material of the metal billet and ring is steel $12 \mathrm{H} 18 \mathrm{~N} 9 \mathrm{~T}$; the geometrical sizes are as follows: $r_{m}=0.015 \mathrm{~m}, r_{l}=0.025 \mathrm{~m}, r_{m l}=0.03 \mathrm{~m}$, $L=0.1 \mathrm{~m}$; the initial billet temperature is $T_{m 0}=840{ }^{\circ} \mathrm{C}$; the initial temperature of the water 
flow is $T_{l 0}=20^{\circ} \mathrm{C}$; the initial temperature of the metal ring is $T_{m l}=T_{l 0}$. The calculations are carried out for the flow velocity a) $u_{0}=0.1 \mathrm{~m} / \mathrm{s}$ and b) $u_{0}=1 \mathrm{~m} / \mathrm{s}$.

Figures $2-4$ show the cooling rate fields in the metal billet volume for the times of cooling $t=1 \mathrm{~s}, t=2 \mathrm{~s}$, and $t=10 \mathrm{~s}$, respectively.

It can be seen that the distributions of the local cooling rates in the billet volume are inhomogeneous having focal character at a smaller rate (Fig. 2a) on the initial area of the contact with the cooling medium. The absolute values of the local rate of the cooling of the billet over the entire volume differ by one order of magnitude. When the velocity of the gas-liquid medium flow increases (Fig. 2b), the pattern of the distribution of the local cooling rates throughout the metal volume becomes more uniform along the section. In this case, the cooling rates have the same order of magnitude along the computational domain length. As the time of cooling increases, it can be seen that the obtained values of the local cooling rate show the general pattern of decreasing.

When the liquid flow velocity is small (Fig. 3a), the localized intensive heat transfer is observed in the metal volume on the area of the initial cooling of the billet. When the velocity of the cooling medium flow is larger (Fig. 3b), the pattern of the billet cooling is characterized by the flattened profile of the lines of the local cooling rate in the metal volume. It can be seen that the cooling front shifts to the billet axis, and the average rate of cooling the metal volume is $100{ }^{\circ} \mathrm{C} / \mathrm{s}$.

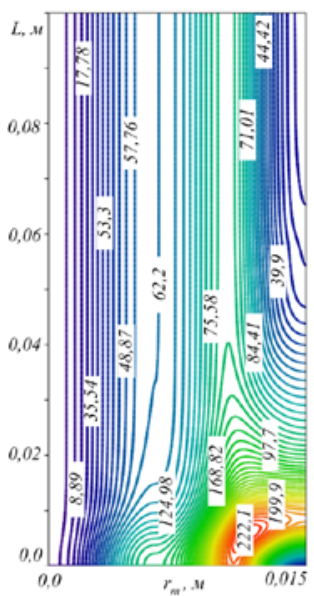

a)

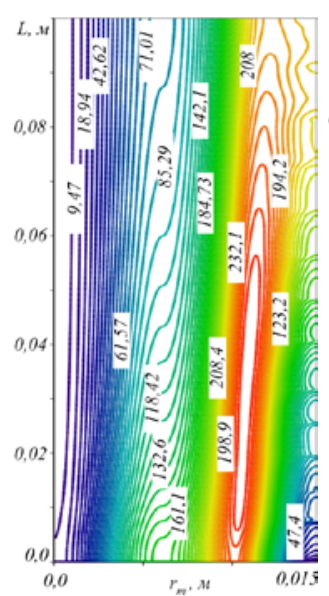

b)

Fig. 2. The billet cooling rate, $t=1 \mathrm{~s}$.

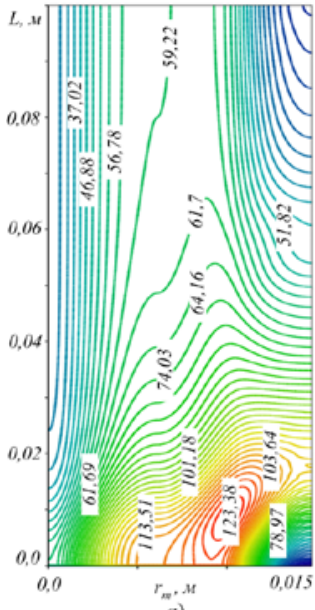

a)

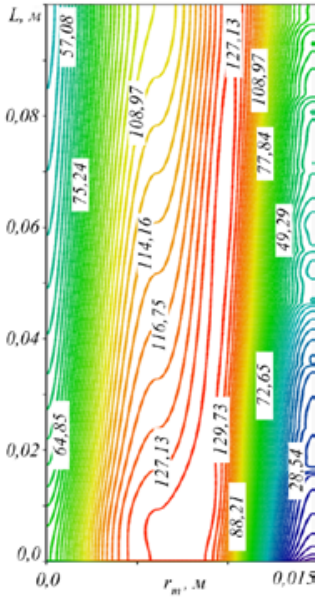

b)

Fig. 3. The billet cooling rate, $t=2 \mathrm{~s}$.

The general tendency of the temperature distribution with time throughout the billet volume for the liquid flow velocities in Fig. $4 \mathrm{a}$ and Fig. $4 \mathrm{~b}$ remains. 


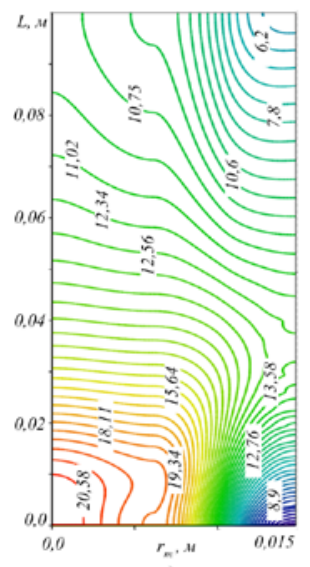

a)

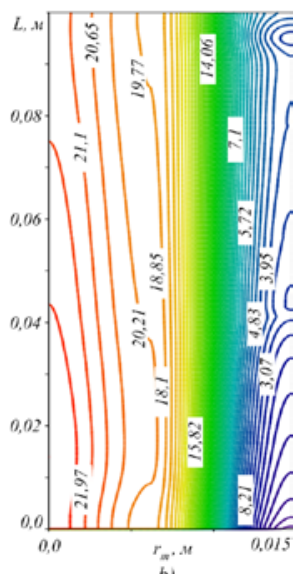

b)

Fig. 4. The billet cooling rate, $t=10 \mathrm{~s}$.

The parametric investigation results for the changing rate of the cooling of the metal billet material at the non-regular mode of cooling allow to predict a forming structure and physical-mechanical properties of material, which is important, for example, for the modes of thermomechanical strengthening of metal billets from structural steels.

\section{Conclusions}

Thus, on the basis of the continuum mechanics approaches, a mathematical model of conjugate heat transfer has been built allowing the conduction of numerical parametric investigations of cooling a high-temperature metal billet from structural steel by a gasliquid medium flow in a vertical circular channel. The investigation results can be used for evaluating a forming structure and physical-mechanical properties of the material of a cooled metal billet.

The work is financially supported by the Complex Program of the Ural Branch of the Russian Academy of Sciences 18-10-1-36

\section{References}

1. Lipanov A.M., Makarov S.S., Karpov A.I., Makarova E.V. Numerical investigation of cooling a high-temperature metal cylinder by a gas-liquid medium flow. Teplofizika i aeromekhanika. Vol. 24, 1, P. 53 - 60. (2017)

2. Makarov S. S., Dement'yev V. B., Makarova E. V. Mathematical modeling of cooling high - temperature cylindrical workpieces. Procedia Engineering, Vol. 150, pp. 393399. (2016)

3. Makarov S.S. Numerical simulation of cooling a metal cylinder by a gas-liquid medium flow moving horizontally in a circular channel. Nauchno-tekhnicheskiy vestnik informatsionnykh tekhnologiy, mekhaniki i optiki. Vol. 17, 2. P. $324-331$. (2017)

4. Makarov S. S., Dement'yev V. B., Makhneva T. M. Numerical simulation of the flow of a gas-liquid medium in a circular channel at cooling a high-temperature metal cylinder with a variable cross-section./ MATEC Web of Conferences. Vol.129, no. 03007,4 p. (2017) 
5. Makarov S.S., Dement'yev V.B. Numerical simulation of heat transfer at cooling a high-temperature metal billet from steel 30HGSN2A. Naukoemkie tekhnologii $\mathrm{v}$ mashinostroenii. 9(75). P. 3 - 8. (2017)

6. Lipanov A. M., Makarov S. S., Karpov A. I. Numerical simulation of the heat transfer at cooling a high-temperature metal cylinder by a flow of a gas-liquid medium. Journal of Physics: Conference Series 891, p. 012036. (2017).

7. Makarov S.S., Lipanov A.M., Karpov A.I. Numerical simulation of conjugate heat transfer at the non-regular mode of cooling a high-temperature metal billet by a gasliquid medium flow in a vertical circular channel. Khimicheskaya fizika i mezoskopiya. Vol. 19. 4. P. 559 - 568. (2017). 